\title{
EK Draconis: Long-term photometry on Sonneberg Sky-Patrol plates
}

\author{
H.-E. Fröhlich, R. Tschäpe ${ }^{\star}$, G. Rüdiger, and K. G. Strassmeier \\ Astrophysikalisches Institut Potsdam, An der Sternwarte 16, 14482 Potsdam, Germany \\ Received 22 January 2002 / Accepted 4 March 2002

\begin{abstract}
We performed long-term photometry of the young solar analog EK Draconis (HD 129333) using 1030 blue-sensitive Sky-Patrol plates taken at Sonneberg Observatory from 1958 onwards. A secular dimming of $0.0057 \pm 0.0008 \mathrm{mag} / \mathrm{y}$ beginning around 1975 was found. Hence, the recent trend first observed with Automatic Photoelectric Telescopes (APTs) is confirmed and traced back to the mid-seventies.
\end{abstract}

Key words. stars: activity - stars: individual: HD 129333 (EK Dra) - stars: late-type - stars: rotation - stars: variables: other

\section{Introduction}

Zeeman-Doppler images of the fast rotating stars $\mathrm{AB}$ Dor (HD 36705) and LQ Hya (HD 82558) reveal that the magnetic field configuration differs essentially from the solar magnetic field (Donati 1999; Jardine et al. 2002). The observed rotation-photospheric-activity correlation (Messina et al. 2001) suggests that one has to distinguish between fast $(P \geq 1 \mathrm{~d})$ and ultrafast $(P<1 \mathrm{~d})$ rotators. This is no surprise. In fast rotating stars differential surface rotation is suppressed, i.e. the relative value of differential rotation is smaller by two to three orders of magnitude than for the slowest rotators (Hall 1991). This, of course, should have bearing on the type of dynamo operating in a fast rotating star. Perhaps such dynamos are not prone to oscillations at all. Anyway, whether fast rotators show activity cycles or not will shed some light on the underlying stellar dynamo.

In the case of LQ Hya $(P=1.6 \mathrm{~d})$ already a cycle period of around 7 years is indicated in photometric data (Strassmeier et al. 1997). Amado et al. (2001) claim for a period of 5.3 years in the light minima of $\mathrm{AB}$ Dor $(P=0.5 \mathrm{~d})$, but this is not yet confirmed. Whether the suggested long-term change in differential surface rotation pattern in AB Dor (Cameron \& Donati 2002) is due to a decade long magnetic cycle, remains to be shown, too.

Here we look for cyclic behaviour in the light of EK Draconis.

The solar analog EK Dra = HD 129333 (Dorren \& Guinan 1994 , and references therein) is a young (probably $70 \mathrm{Myr}$ ) fast rotating single ${ }^{1}$ star with spot induced brightness variations up

Send offprint requests to: $\mathrm{H}$.-E. Fröhlich, e-mail: HEFroehlich@aip.de

* R. Tschäpe past away in April 2002.

1 There is some indication that EK Dra may belong to a very-longperiod binary system (Duquennoy \& Mayor 1991). to 0.09 mag. The inclination angle is $\approx 60^{\circ}$ (Strassmeier \& Rice 1998). The photometric period ranges from 2.55 to 2.80 days (DePasquale et al. 2001). Its variation hints at differential latitudinal rotation. EK Dra is very active with chromospheric emission levels 3-5 times solar levels (Baliunas et al. 1995), with coronal X-ray emission 400 times higher than the quiet sun (Dorren \& Guinan 1994; Dorren et al. 1995; Güdel et al. 1995b; Güdel et al. 1997), with coronal microwave emission (Güdel et al. 1995a). It shows frequent flares (Audard et al. $1999)$ in the EUV and several cool spots. The dominating feature is a spot at a latitude of $\approx 70^{\circ}-80^{\circ}$ (Strassmeier \& Rice 1998).

There are already indications for an activity cycle of 12 years in the mean light of this star (Dorren \& Guinan 1994; Bochanski et al. 2000; DePasquale et al. 2001; Messina \& Guinan 2001). (But note that no such cycle is seen in the data from Radick et al. 1998.) This undulation is superimposed upon a secular decline discernable in the robotic telescope data from Strassmeier et al. (1997, 1999) and Messina \& Guinan (2001). Such a trend hints at considerable variability on a much longer time scale. Even an abrupt level shift by $\approx 0.05 \mathrm{mag}$ around 1988 has been reported (Lockwood et al. 1997). In order to study the star's long-term behaviour one has to fall back upon photographic observations. Sky-patrol surveys where the same region of the sky is recorded regularly under comparable conditions are very aptly.

Long-term photographic photometry of other BY Dra variables using archival plate collections has already revealed remarkable amplitudes up to $1.0 \mathrm{mag}$ (PZ Mon) with time-scales of 30-60 years in the case of active red dwarfs (Phillips \& Hartmann 1978; Hartmann et al. 1979; Hartmann et al. 1981; Bondar' 1995; Alekseev \& Bondar' 1998). These early investigations have been done by visual inspection of plates with magnifying glasses or iris photometry, respectively. 
Here we extend these studies to a very active solar-type star, using CCD line scans of more than thousand Sky-patrol plates and restricting if possible to bona fide comparison stars of spectral type A and F thought to be photometrically much more reliable than late-type stars.

\section{Observations}

In 1958 a modernized sky-patrol program with short-focus cameras monitoring the whole visible sky and especially designed to support long-term studies of variable stars has been commenced at Sonneberg Observatory (Bräuer \& Richter 1994). From that year onwards more than 1400 B plates of the four patrol fields (with centre positions: RA = $13^{\mathrm{h}}, 14^{\mathrm{h}}, 15^{\mathrm{h}}$, and $\left.16^{\mathrm{h}}, \delta=60^{\circ}\right)$ containing EK Dra have been obtained. (Additionally, there are approximately $500 \mathrm{~V}$ plates of these fields waiting to be scanned and analyzed.) The photographic plates cover $26^{\circ} \times 26^{\circ}$ on the sky up to a limiting magnitude of $\approx 14^{\mathrm{m}}$. From each plate a small rectangular area of $\approx 10$ square degrees containing EK Dra and six fiducial stars has been scanned by means of an 8-bit CCD line scanner with $15 \mu \mathrm{m}$ resolution, corresponding to $12.4 \mathrm{arcsec} / \mathrm{pxl}$ (Kroll \& Neugebauer 1993).

\section{Long-term photographic photometry}

Longitudinal studies almost always lack homogeneity and comparability of the data. Neither the photographic photometry has been done on photographs taken on identical emulsions, nor these emulsions have been developed together. Over the span of years photographic plates, even if labeled as B plate for instance, differ inevitably in colour sensitivity due to differing emulsions used, changes in the plate processing procedure and so on. There are other issues encountered, too:

- The mean zenith distance of the EK-Dra region varies over the years in a systematical manner by $\approx 18^{\circ}$;

- Only one sky-patrol field, that with centre $\left(14^{\mathrm{h}}, 60^{\circ}\right)$, covers the full time span. With 700 plates it is the most extensive. Exposures of the other three fields are mainly from the early sixties;

- The sizes of the stellar images have shrunk by 15 per cent over the span of 35 years.

In order to cope with such effects we can only rely on the presumed constancy of the fiducial stars. The $B_{\mathrm{T}}$ and $V_{\mathrm{T}}$ brightnesses of these stars are taken from the Tycho- 2 catalogue (Høg et al. 2000a; Høg et al. 2000b). The photometric system differs slightly from Johnson's. For main-sequence stars the approximation $V=V_{\mathrm{T}}-0.090(B-V)_{\mathrm{T}},(B-V)=0.850(B-V)_{\mathrm{T}}$ holds (Perryman 1997).

After having performed a preliminary analysis of our data we became aware that restricting to one camera type (55/2500 Tessar) improves the smoothness of our light curves considerably. We have therefore excluded all the plates from our sample not taken with that kind of camera.
Table 1. $B_{\mathrm{T}}$ and $(B-V)_{\mathrm{T}}$ for the stars measured. Data are from the Tycho-2 catalogue (Høg et al. 2000b). Additionally, spectral types from the SAO Star Catalog are given (EK Dra's spectral type is taken from Strassmeier et al. 1997).

\begin{tabular}{cccl}
\hline \hline $\mathrm{HD}$ & $B_{\mathrm{T}}$ & $(B-V)_{\mathrm{T}}$ & Sp. \\
\hline 128492 & $8.905 \pm 0.017$ & 1.329 & $\mathrm{~K} 2$ \\
128815 & $8.520 \pm 0.016$ & 0.409 & $\mathrm{~A} 3$ \\
129333 & $8.385 \pm 0.016$ & 0.708 & $\mathrm{G} 1.5$ \\
129390 & $8.019 \pm 0.016$ & 0.427 & $\mathrm{~F} 2$ \\
130460 & $7.793 \pm 0.015$ & 0.521 & $\mathrm{~F} 5$ \\
130600 & $8.107 \pm 0.015$ & -0.092 & A0 \\
130914 & $8.037 \pm 0.015$ & 0.292 & A5 \\
\hline
\end{tabular}

\subsection{Outline of the method}

A single brightness estimate on a scanned sky-patrol plate is accurate to 0.07-0.1 mag (Vogt \& Kroll 1999). By averaging over $N$ estimates one can hope to reduce the photometric error according to the $N^{-1 / 2}$-law, provided one harnesses all kinds of systematic errors inherent in long-term photographic photometry. Rotationally induced short-term brightness variation is considered here as an additional random noise.

We are looking for a brightness estimator, an interpolation recipe, with some physical background, i.e. a procedure exploiting sort of parameterized characteristic curve in order to convert - at least approximately - measured photographic densities into intensities, and which ultimately let the brightnesses of the fiducial stars unaltered over the course of time.

\subsection{Fiducial stars}

Up to six fiducial stars were used for interpolation. For homogeneity's sake only stars with entries in the Tycho catalogue have been chosen (see Table 1). The stars span the range from 7 . $8 \leq B_{\mathrm{T}} \leq 8$. 9 , enclosing EK Dra's mean magnitude $\left(B_{\mathrm{T}} \approx 8.4\right)$ very well. With regard to colour, the situation is sort of worse. Only HD 128492, a K2 giant, is redder than EK Dra. Despite its late spectral type this star has been tentatively included to enlarge the range of colours beyond EK Dra's. Unfortunately, the reddest fiducial star is also the weakest, hence introducing a correlation in the $B_{\mathrm{T}}-(B-V)_{\mathrm{T}}$ plane of the fiducial stars. The analysis have been done therefore twice, with HD 128492 included or omitted, respectively.

All other fiducial stars belong to spectral types $\mathrm{A}$ and $\mathrm{F}$ and are therefore bona fide constant. But, we know this for sure only in the case of HD 129390. This star is next to EK Dra and its quoted long-term constancy is $\pm 0.0008 \mathrm{mag}$ (Lockwood et al. 1997).

\subsection{Parameter fitting}

The sky-patrol plates are uncalibrated. Inevitable changes in the colour sensitivity alone make the blackness of a star unsuited as brightness indicator in long-term photometric studies. Our approach uses therefore a parameterized characteristic curve in order to turn photographic densities into intensities. Before doing this the 8-bit readings from the CCD scanner, 
which are proportional to transparency, had to be converted into densities, with the blackest pixel in the scanned area being assigned infinite photographic density.

After experimenting with a two-parameter representation of the characteristic curve which leads to a far to high mock accuracy and a strong correlation between the two parameters (gradation and a non-linear term) we decited to neglect any non-linearity and to fix the gradation, $\gamma=1$, confirming Kroll $\&$ Neugebauer (1993) who claimed that this already yields, after background subtraction and integration within a centred aperture, satisfying photometric results.

Intensity estimates like that are referred to the local sky background. The mode of the frequency distribution of intensity in a $50 \mathrm{pxl} \times 50 \mathrm{pxl}$ field around each star has been assigned arbitrarily the value unity. After background subtraction and integrating over all pixels within the circular aperture, one gets the sought flux and magnitude estimate. A two-dimensional Gauss fit in intensity space provides besides the exact centering a star's diameters along the major and the minor axes and the position angle of the major axis. While the results from point-by-point integrations within an appropriately fixed circular aperture are in agreement with flux estimates from twodimensional Gauss fits, the fluxes from Gauss fits, utilizing the marginal distributions in both the $x$ - and $y$-directions only, are generally overestimated because the stellar images lack circular symmetry.

In order to tie magnitudes into the Tycho- 2 photometric system one has to apply time-dependent colour corrections. Here we restrict ourselves to a model (see Eq. (1)) where the coefficients in the colour equation depend linearly on time - a rather conservative approach introducing at the most spurious trends, never periodicities.

Besides a drift in colour sensitivity we have made allowance for a field error. Here we have exploited the fact, that the fiducial stars lie accidentally on a rather straight line. Because the distances between the fiducial stars are small as compared with the plate size we are allowed to describe the field error by merely a linear dependence on one coordinate, giving cause for four further parameters because each of the four sky fields, differing in hour angle, has to be assigned its own field correction.

Differential extinction, a minor correction, is accounted for, too. Neglecting the curvature effect we have taken the prescription given by Allen (1976).

Altogether, we have to fix five free parameters, where one depends on its part on the hour angle of the plate centre:

$$
\begin{aligned}
B_{i}= & B_{i}^{\text {plate }}+\left(p_{1}+p_{2} \cdot\left(t_{\mathrm{a}}-1975\right)\right) \cdot(B-V)_{i}^{\mathrm{T}} \\
& +\left(p_{3}+p_{4} \cdot\left(t_{\mathrm{a}}-1975\right)\right) \cdot\left((B-V)_{i}^{\mathrm{T}}\right)^{2} \\
& +p_{5 \mathrm{a} . . .5 \mathrm{~d}} \cdot \Delta x_{i}+\text { const. },
\end{aligned}
$$

where $t_{\mathrm{a}}$ denotes the year, $\Delta x_{i}$ measures a star's distance to EK Dra, and the offset const is a mere nuisance parameter for each plate. Variables labeled with a superscript "T" refer to Tycho-2 values. Our model is not only linear in time, but linear in its parameters, too.
We note, that we have tried further free parameters too. The additional effects considered were a scale error and the Purkinje effect, but the gain in goodness-of fit was only marginal.

We are looking for a compromise between conflicting aims of fidelity. Both, the residuals should be as small as possible and the long-term linear trends of the fiducial stars should be insignificant. Hence, we chose the following prescription: the "best" set of parameters for the whole sample minimize the mean of the sum of the quadratic residuals for the six fiducial stars, subject to the constraint that the largest linear trend (the slope if one performs a regression analysis) in the ensemble of fiducial stars is not significant at a one sigma level:

$$
\left\langle\frac{1}{6} \sum_{i=1}^{6}\left(B_{i}-B_{i}^{T}\right)^{2}\right\rangle+\lambda \cdot \max \left(|s| / \sigma_{\mathrm{s}}\right)=\text { Min. }
$$

where $s$ denotes the slope, $\sigma_{\mathrm{s}}$ its standard deviation. The regularization parameter $\lambda$ has been varied until $\max \left(|s| / \sigma_{\mathrm{s}}\right) \approx 1$.

The parameter values, $p_{1} \ldots p_{5 \mathrm{~d}}$, found by these constrained least-squares are merely the most probable estimates among many others which were also acceptable. If the posterior-probability distribution in the parameter space is somewhat skewed such a maximum likelihood estimate becomes biased. Briefly put, one should prefer mean values. But this would mean exploring and integrating over the eightdimensional parameter space which is computationally prohibitive time-consuming.

For a given sky-patrol field we have fitted altogether five free parameters. The question arises how significant are these parameters, are they really needed? The answer depends whether you share a Bayesian view or not. Taking the classical view we are allowed to fit a lot of parameters because we have lots of data. Then even a modest gain in goodness-of-fit proves significant, i.e. exceeds the gain due to the mere enlargement of the number of degrees of freedom.

\section{Results}

\subsection{Constancy of fiducial stars}

Exploiting 1030 B plates of the Sonneberg Sky-patrol survey, we are able to draw within the limitations of our linear model EK Dra's past light-curve over 35 years (see Fig. 1). Each time bin comprises brightness estimates from about 50 plates. Shown are weighted averages. Weights are according to a plate's estimated photometric accuracy. The error bars indicate standard deviations of these weighted bin means.

In order to check the constancy of the fiducial stars, two statistical tests have been performed, an analysis of variance and Friedman's test. Until now the photometric zero point on each plate has been fixed by requiring the deviations from the Tycho- 2 brightnesses to cancel out. Now, in order to remove the interdependencies due to this procedure, we refer to the measured mean brightness of HD 129390 and HD 130914, the two stars most similar in the colour-magnitude diagram. In the forthcoming statistical analyses we have to discard both stars. While the situation is somewhat changed, we are convinced that the test results are applicable to the proper case 

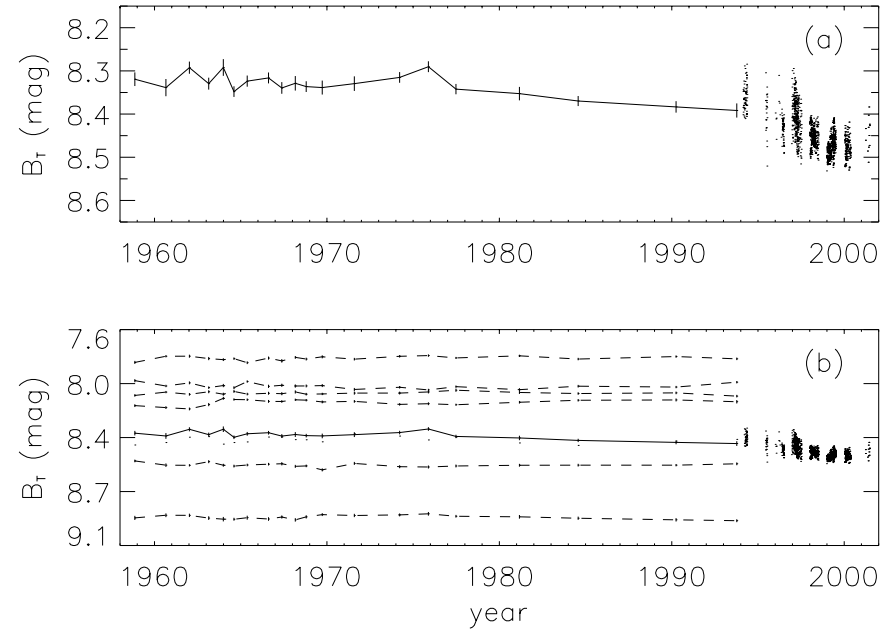

Fig. 1. $B_{\mathrm{T}}$ light-curves: a) EK Dra, b) EK Dra (full line) and six fiducial stars. From 1994 onwards EK Dra has been monitored by APTs (Strassmeier et al. 1997; Strassmeier et al. 1999, and yet unpublished measurements). These photo-electrically measured brightnesses, not averaged, are shown too.

where the light curves are referred to the centre of gravity of the brightness of all fiducial stars.

We have divided the time span into 17 time bins, each bin containing up to 52 measurements. A variance analysis of the light curves of the remaining four fiducial stars reveals then the following. The average (weighted) error of a brightness estimate amounts to $\approx 0.085 \mathrm{mag}$. In the quadratic mean a fiducial star is off by 0.014 mag from its Tycho- 2 brightness, which is within the quoted catalogue's photometric accuracy of $0.015 \ldots 0.017 \mathrm{mag}$. Besides, there are long-term excursions (i.e. from one bin to another one) of $0.010 \mathrm{mag}$ in the brightnesses of the fiducial stars (significant at a one per cent level) our linear model cannot cope with. Refinements of the fitting procedure beyond the linear model are of course imaginable, but - as at the expense of reliability - not really desirable.

Tests on variances are known to depend strongly on the assumption of normality. Concededly, while in most time bins a Gaussian distribution of the estimated brightnesses proves justified, there are significant deviations from normality in others. Therefore, we have performed Friedman's test too, which is a rank test and as such free from whatsoever the underlying distribution is. This test certifies us that (at a five per cent level) the factor time does not affect the data.

\subsection{EK Dra's long-term trend}

In the upshot EK Dra's blue brightness declines by $0.0017 \pm$ $0.0004 \mathrm{mag} / \mathrm{y}$ for the whole time span of 35 years. From 1975 onwards the trend is even more pronounced, viz. $0.0057 \pm$ $0.0008 \mathrm{mag} / \mathrm{y}$. If this is due to an activity cycle, the period must exceed half a century, the amplitude at least 0.04 mag. An even stronger fading of $\simeq 0.007 \mathrm{mag} / \mathrm{y}$ is indicated for the last 15 years (Messina \& Guinan 2001). Superimposed on this long-term trend is a variation with a period of 11 years and an amplitude of $\approx 0.05 \mathrm{mag}$. Because of our coarse binning for that time span we are unable to confirm such a period.
EK Dra's significant (4.7- $\sigma$ effect) long-term fading does not depend on whether the somewhat suspicious K2 star is included or not. Without HD 128492 the trend were significant, too $(0.0014 \pm 0.0004 \mathrm{mag} / \mathrm{y})$. In order to make an appropriate colour correction its inclusion proves necessary.

Furthermore, we have tried two aperture radii, 7.5 pixel and 15 pixel, respectively. There are no differences in the results, worth speaking of.

Repeating the analysis, this time with EK Dra considered constant too, does not result in a satisfying fit at all. Even if one were willing to tolerate a bi-modal distribution of the residuals, their standard deviation would exceed $0.2 \mathrm{mag}$, which is beyond all current experience.

In the case of EK Dra the average standard deviation from a bin mean systematically exceeds the estimated mean photometric accuracy by a factor of $\approx 1.5$. This implies an additional short-term variability of $0.08 \mathrm{mag}$. There is no indication that the strength of this obviously spot induced rotational modulation depends on time.

\section{Discussion and conclusions}

Using their empirical correlations between dimensionless cycle length and the inverse Rossby number, Saar \& Brandenburg (1999) tried to predict the cycle length for young stars in open clusters. In the case of EK Dra they give putative cycle lengths of 1.4 and 39 years, depending whether they locate EK Dra on the "active" branch or the "superactive" one, respectively. But, because there are a lot of transition stars between these two branches (albeit at somewhat larger inverse Rossby numbers) a cycle length below 39 years is not excluded from the outset. The observed "ripples" in the Ca-II data (Baliunas et al. 1995) as well as in the photometric data (Radick et al. 1998) were reconcilable with EK Dra belonging to the "active" branch, the long-term trend confirmed by our photometry hints at the "superactive" branch. Perhaps EK Dra belongs to both, exhibiting a superposition of two modes.

From a theoretical point of view there are two completely different solutions of the mean-field dynamo equation in dependence on the strength of the non-uniformity of the internal rotation law. If - as for the Sun - the differential rotation is strong then the resulting magnetic field is axisymmetric and oscillating, with a dominating toroidal field component (Steenbeck \& Krause 1968). As far as we know the cycle times are of the order of years or of decades. However, it is not yet known whether longer periods exist (for a brief literature summary see Saar \& Brandenburg 1999; Oláh et al. 2000). Maunder minima are possible due to the nonlinear interaction between flow and magnetic field. On the other hand, for uniform (or nearly uniform) basic rotation the solution of the dynamo equation for stars with thin or thick convection zone is strongly differing. There is no turbulence model resulting in a scalar $\alpha$-effect. All the calculations and simulations provide the $\alpha$-effect as a tensor with components which are not only differing in their amplitudes but even in their signs (Ferrière 1992a; Ferrière 1992b; Rüdiger \& Kichatinov 1993; Ziegler et al. 1996). Then the resulting large-scale magnetic fields are symmetric with respect to the equator but not symmetric with respect to the rotation axis 
(Rüdiger 1980; Rüdiger \& Elstner 1994; Moss \& Brandenburg 1995). These fields are (slowly) drifting in longitude but they are never oscillating. Producing oscillating magnetic modes in the absence of differential rotation is not a simple task. One has to concentrate the (anisotropic) $\alpha$-effect to the equatorial region (Elstner et al. 2002). The Zeeman-Doppler images for AB Dor $\left(P_{\text {rot }}=12.5 \mathrm{~h}\right)$ and LQ Hya $\left(P_{\text {rot }}=1.6 \mathrm{~d}\right)$ seem to support these finding (Donati 1999; Jardine et al. 2002). The field geometry for fast rotating main sequence stars is unlike the solar one.

Acknowledgements. This work were not been possible without the generous support by Peter Kroll from Sonneberg Observatory. KGS acknowledges support from DFG grants STR645/1 and HU 532/8. The referee is thanked for valuable suggestions improving the paper.

\section{References}

Alekseev, I. Y., \& Bondar', N. I. 1998, Astron. Rep., 42, 655

Allen, C. W. 1976, Astrophysical Quantities, 3rd edition (London: Athlone)

Amado, P. J., Cutispoto, G., Lanza, A. F., \& Rodonò, M. 2001, in ASP Conf. Ser. 223, 11th Cambridge Workshop on Cool Stars, Stellar Systems and the Sun, ed. R. J. García López, R. Rebolo, \& M. R. Zapatero Osorio, 895

Audard, M., Güdel, M., \& Guinan, E. F. 1999, ApJ, 513, L53

Baliunas, S. L., Donahue, R. A., Soon, W. H., et al. 1995, ApJ, 438, 269

Bochanski, J. J., DePasquale, J. M., DeWarf, L. E., et al. 2000, Am. Astron. Soc. Meet., 196, 4607

Bondar', N. I. 1995, A\&AS, 111, 259

Bräuer, H.-J., \& Richter, G. A. 1994, in IAU Symp. 161, Astronomy from Wide-Field Imaging, ed. H. T. MacGillivray, E. B. Thomson, B. M. Lasker, et al. (Kluwer Academic Publishers, Dordrecht), 43 Cameron, A. C., \& Donati, J.-F. 2002, MNRAS, 329, L23

DePasquale, J. M., Bochanski, J. J., Guinan, E. F., et al. 2001, Am. Astron. Soc. Meet., 198, 4604

Donati, J.-F. 1999, MNRAS, 302, 457

Dorren, J. D., Güdel, M., \& Guinan, E. F. 1995, ApJ, 448, 431

Dorren, J. D., \& Guinan, E. F. 1994, ApJ, 428, 805

Duquennoy, A., \& Mayor, M. 1991, A\&A, 248, 485

Elstner, D., et al. 2002, in prep.

Ferrière, K. 1992a, ApJ, 389, 286
Ferrière, K. 1992b, ApJ, 391, 188

Güdel, M., Guinan, E. F., Mewe, R., Kaastra, J. S., \& Skinner, S. L. 1997, ApJ, 479, 416

Güdel, M., Schmitt, J. H. M. M., \& Benz, A. O. 1995a, A\&A, 302, 775

Güdel, M., Schmitt, J. H. M. M., Benz, A. O., \& Elias, N. M. 1995b, A\&A, 301, 201

Hall, D. S. 1991, in The Sun and Cool Stars, Activity, Magnetism, Dynamos. Proceedings of Colloquium No. 130 of the International Astronomical Union, held in Helsinki, Finland, July 17-20, 1990, ed. I. Tuominen, D. Moss, \& G. Rüdiger (SpringerVerlag, Berlin, New York), 353

Hartmann, L., Dussault, M., Noah, P. V., Klimke, A., \& Bopp, B. W. 1981, ApJ, 249, 662

Hartmann, L., Londono, C., \& Phillips, M. J. 1979, ApJ, 229, 183

Høg, E., Fabricius, C., Makarov, V. V., et al. 2000a, A\&A, 357, 367

Høg, E., Fabricius, C., Makarov, V. V., et al. 2000b, A\&A, 355, L27

Jardine, M., Collier Cameron, A., \& Donati, J.-F. 2002, MNRAS, 333, 339

Kroll, P., \& Neugebauer, P. 1993, A\&A, 273, 341

Lockwood, G. W., Skiff, B. A., \& Radick, R. R. 1997, ApJ, 485, 789

Messina, S., \& Guinan, E. F. 2001, in 12th Cambridge Workshop on Cool Stars, Stellar Systems and the Sun, The Future of Cool-Star Astrophysics, poster 06.07, in press

Messina, S., Rodonò, M., \& Guinan, E. F. 2001, A\&A, 366, 215

Moss, D., \& Brandenburg, A. 1995, Geophys. Astrophys. Fluid Dyn., 80,229

Oláh, K., Kolláth, Z., \& Strassmeier, K. G. 2000, A\&A, 356, 643

Perryman, M. A. C. 1997, ESA SP-1200, 120

Phillips, M. J., \& Hartmann, L. 1978, ApJ, 224, 182

Radick, R. R., Lockwood, G. W., Skiff, B. A., \& Baliunas, S. L. 1998, ApJS, 118, 239

Rüdiger, G. 1980, Astron. Nachr., 301, 181

Rüdiger, G., \& Elstner, D. 1994, A\&A, 281, 46

Rüdiger, G., \& Kichatinov, L. L. 1993, A\&A, 269, 581

Saar, S. H., \& Brandenburg, A. 1999, ApJ, 524, 295

Steenbeck, M., \& Krause, F. 1968, Astron. Nachr., 291, 49

Strassmeier, K. G., Bartus, J., Cutispoto, G., \& Rodono, M. 1997, A\&AS, 125, 11

Strassmeier, K. G., \& Rice, J. B. 1998, A\&A, 330, 685

Strassmeier, K. G., Serkowitsch, E., \& Granzer, T. 1999, A\&AS, 140, 29

Vogt, N., \& Kroll, P. 1999, Acta Hist. Astron., 6, 210

Ziegler, U., Yorke, H. W., \& Kaisig, M. 1996, A\&A, 305, 114 